\title{
Die Rolle der Digitalisierung bei der Transformation vom Produzenten zum produzierenden Dienstleister
}
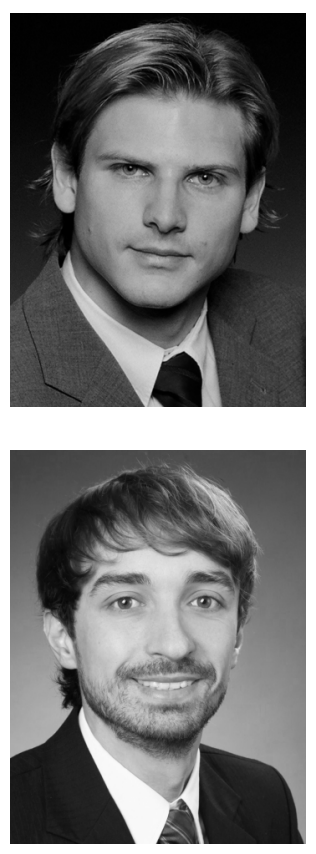

\section{Christian Lerch und Matthias Gotsch}

Produktbegleitende Dienstleistungen; Industrielle Dienstleistungen; Dienstleistungstypologie; Digitalisierung; Produkt Service System; Digitales Produkt Service System

Product-related services; Industrial Services; Service typology; Digitalization; Product service system; Digital product service system

Klassische Rahmenmodelle für industrielle Dienstleistungen sind zwar in der Lage, die zunehmende Tertiarisierung vieler bedeutender Branchen des Verarbeitenden Gewerbes abzubilden, können aber gleichzeitig nicht in ausreichender Intensität die zunehmende Digitalisierung vieler industriell angebotener Dienstleistungen berücksichtigen. Durch das Verschmelzen der beiden Trends - Tertiarisierung und Digitalisierung - ergeben sich jedoch beträchtliche Auswirkungen, die unter anderem die Transformation vom Produzenten zum produzierenden Dienstleister tangieren und diese zeitlich beschleunigen können. Daher entwickelt dieser Beitrag ein neues Transformationsmodell, das unter Berücksichtigung der beiden Trends ein neues Bezugssystem für das Dienstleistungsangebot von Produktherstellern entwirft.

Classic frames models for industrial services are indeed able to map the growing servitization of many important manufacturing industries, but cannot take into account sufficiently the increasing digitalization of many industrially offered services simultaneously. By fusing the two trends - servitization and digitalization - there are significant implications, which affect the transformation from a producer to a manufacturing service provider and even significantly speed up. Therefore, this paper develops an attempt to establish a new transformation model, taking into consideration the two trends, creating a new reference system for offering services of manufacturers.

\section{Einleitung}

In den letzten Jahren hat der Trend der so genannten Tertiarisierung eingesetzt, der eine verstärkte Dienstleistungsorientierung in der Industrie beschreibt (Gerhardt 2000). Produkthersteller verkaufen nicht mehr ihre Produkte, sondern beginnen zusätzlich auf das Produkt abgestimmte Dienstleistungen anzubieten (Vandermerwe/Rada 1988; Wise/Baumgartner 1999; Baines et al. 2009). Diese reichen von traditionellen produktbegleitenden Dienstleistungen, wie z.B. Wartung, Reparatur und Schulung, bis hin zu avancierten kun- 
denorientierten Leistungsbündeln (Mathieu 2001; Oliva/Kallenberg 2003; Lay 2014), welche materielle und immaterielle Leistungskomponenten beinhalten (Engelhardt et al. 1993).

Durch diese industrielle Entwicklung wird davon ausgegangen, dass sich Produkthersteller auf einem Transformationspfad befinden und sich kontinuierlich über die Zeit vom reinen Produzenten zum Anbieter innovativer Dienstleistungen wandeln (Gebauer 2004; Gebaner et al. 2005). Dieser Transformationspfad wird in der Literatur in verschiedenen Beiträgen analysiert und mit unterschiedlichen Stufen und strategischen Entwicklungspotenzialen beschrieben (More 2001; Oliva/Kallenberg 2003; Penttinen/Palmer 2007; Gebauer et al. 2008; Matthyssens/Vandenbempt 2008; Matthyssens/Vandebempt 2010). Am Ende des Pfads bieten Hersteller innovative Produkt Service Systeme wie z.B. Verfügbarkeitsgarantien oder Betreibermodelle an, die einerseits den Kundennutzen erhöhen und andererseits neue Wertschöpfungspotenziale für den Anbieter eröffnen (Boyt/Harvey 1997; Brady et al. 2005).

Jenseits des Trends der Tertiarisierung beginnen Produzenten außerdem, ihre Produkte mit intelligenten Systemen auszustatten, damit Maschinen und Anlagen in der Produktion miteinander kommunizieren und eigenständig agieren können. Diese Entwicklung wird auch als Industrie 4.0 bezeichnet, welche die Digitalisierung traditioneller Industrien umfasst (Spath et al. 2013; Bauer et al. 2014). Als Konsequenz aus dieser Entwicklung beginnen Produkthersteller nun auch verstärkt digitale Systeme für ihre Dienstleistungsangebote einzusetzen (Münster/Meiren 2011). So wird bereits seit einigen Jahren die digitale Unterstützung zur Erbringung von Dienstleistungen unter dem Begriff des Teleservice zusammengefasst (Borgmeier 2002).

Durch die weitreichenden Möglichkeiten, welche der Trend der Digitalsierung Produktherstellern bietet, stellt sich die Frage, inwieweit digitale Systeme den Wandel vom Produzenten zum Lösungsanbieter beeinflussen, welche Veränderungen sich hierdurch bei der Transformation ergeben und welche Formen einer Digitalisierung industrieller Dienstleistungen jenseits des klassischen Teleservice existieren. Um diese Fragestellungen zu analysieren, untersucht dieser Beitrag mit Hilfe dreier Fallstudien, wie Produkthersteller digitale Systeme für ihre Dienstleistungsangebote einsetzen und welche Ziele damit verfolgt werden. Hieraus lassen sich Wechselwirkungen beider Trends auf betrieblicher Ebene identifizieren, die dann auf bestehende Transformationsmodelle übertragen werden. Dadurch lässt sich ein Rahmenkonzept schaffen, welches die Transformation von Produzenten unter dem Einfluss von Digitalisierung und Tertiarisierung beschreibt. Der Beitrag schließt mit einem Ausblick und zukünftigem Forschungsbedarf.

\section{Produkthersteller unter dem Einfluss von Tertiarisierung und Digitalisierung}

2.1 Transformation vom Produzenten zum Dienstleistungsanbieter

Die zunehmende Bedeutung von Dienstleistungen in der Industrie und die damit verbundene Tertiarisierung vieler Branchen ist als Trend bereits vor geraumer Zeit erkannt worden und wurde in der Literatur auch ausführlich diskutiert (Vandermerwe/Rada 1988; Wise/ Baumgartner 1999; Baines et al. 2009). Unterschieden werden dabei meist produktbegleitende Dienstleistungen, wie z.B. Wartung, Reparatur oder Schulungen (Schniering 2009), sowie avanciertere Produkt Service Systeme, wie z.B. Verfügbarkeitsgarantien oder Betreibermodelle (Mathieu 2001; Oliva/Kallenberg 2003). 
Definitionsgemäß werden industrielle Dienstleistungen von produzierenden Unternehmen angeboten und von anderen produzierenden Unternehmen nachgefragt (vgl. Abbildung 1). Zudem lassen sich neben einem Produzenten, der ausschließlich obligatorische Dienstleistungen anbietet, zwei weitere Typen von Dienstleistungsanbietern identifizieren (Spath/Demuß 2003). Zum einen der so genannte dienstleistende Produzent, der z.B. um die Verfügbarkeit seiner Produkte zu erhöhen produktbegleitende Dienstleistungen anbietet, und zum anderen der produzierende Dienstleister, der insbesondere lösungsgetrieben agiert und daher so genannte lösungsorientierte Dienstleistungen anbietet, wie z.B. Betreibermodelle (vgl. auch Gebauer 2004).

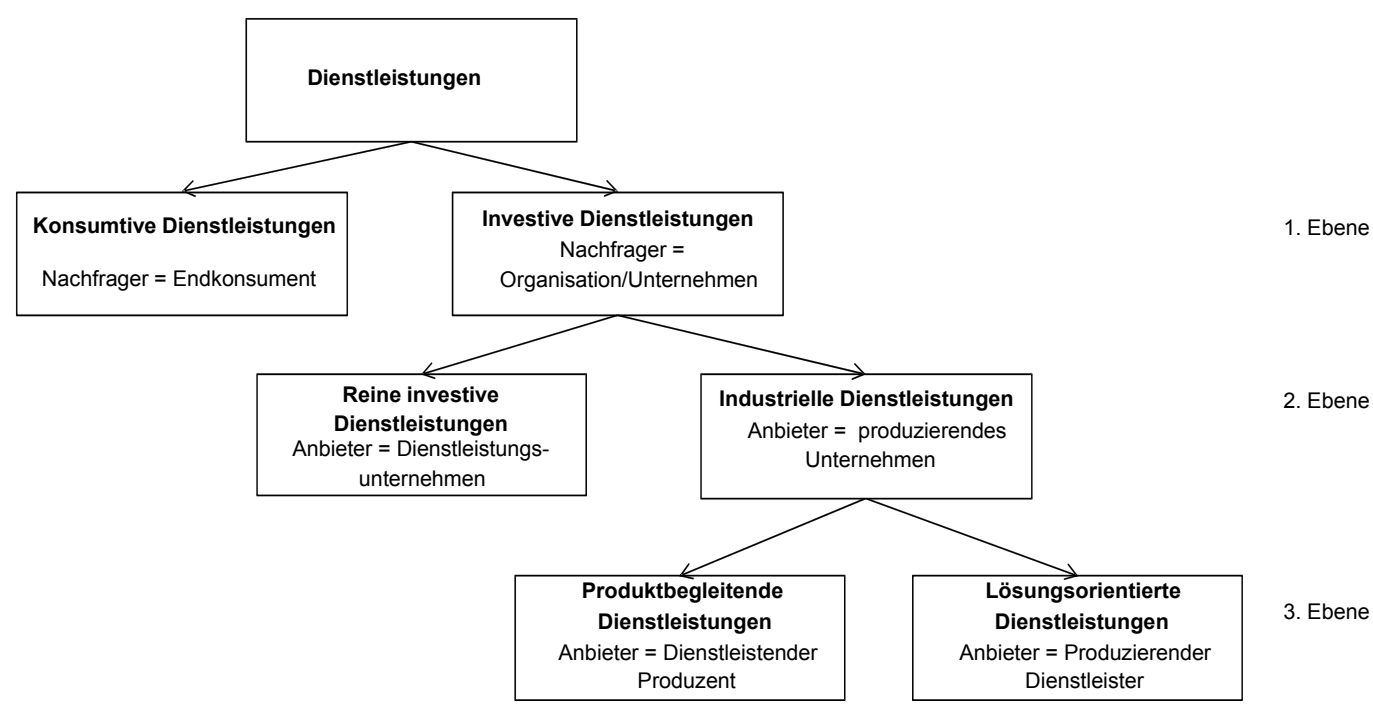

Abbildung 1: Definition industrieller Dienstleistungen (Spath/Demuß 2003)

Diese Aufteilung lässt sich ebenso anhand des Grads der Materialität der Produkte nachvollziehen. So können die vom produzierenden Unternehmen angebotenen Produkte hinsichtlich ihrer materiellen und immateriellen Leistungskomponenten aufgetrennt und anhand des steigenden Immaterialitätsgrades der Leistung in eine Skala eingeordnet werden. Eine weit verbreitete Einteilung bezüglich der Produkt-, Prozess-, und Ergebnisebene industrieller Dienstleistungen findet sich bei Tukker (2004). In dieser Typologie werden drei archetypische Kategorien von Produkt Service Systemen vorgestellt (vgl. Abbildung 2). Die erste Kategorie, produkt- und funktionsorientierte Dienstleistungen, besteht insbesondere aus Beratungsleistungen sowie aus der Pflege und Versorgung mit Verbrauchsmaterialien. Solche Dienstleistungen werden zumeist am Produktverkauf ausgerichtet und stellen somit die traditionelle und meist verbreitete Herangehensweise der verarbeitenden Industrie dar. Die zweite Kategorie der verfügbarkeits- und nutzenorientierten Dienstleistungen umfasst z.B. das Leasing sowie die Vermietung und Bündelung mit Produkten, die jedoch im Eigentum des Herstellers verbleiben. In dieser Kategorie nutzt der Kunde das Produkt und zahlt für seine Verwendung. Die dritte Kategorie, bedürfnis- und ergebnisorientierte Dienstleistungen, stellt den weitestgehenden Schritt der Dienstleistungsintegration dar. In dieser Kategorie hat der Kunde nicht für die Verwendung des Produktes zu zahlen, son- 
dern seine Zahlungshöhe richtet sich vielmehr nach den erzielten Ergebnissen. Damit wird der Hersteller mehr oder weniger zum Outsourcing-Partner seiner Kunden und garantiert ihnen sowohl Ergebnis als auch Qualität der Leistung (Lay 2014).

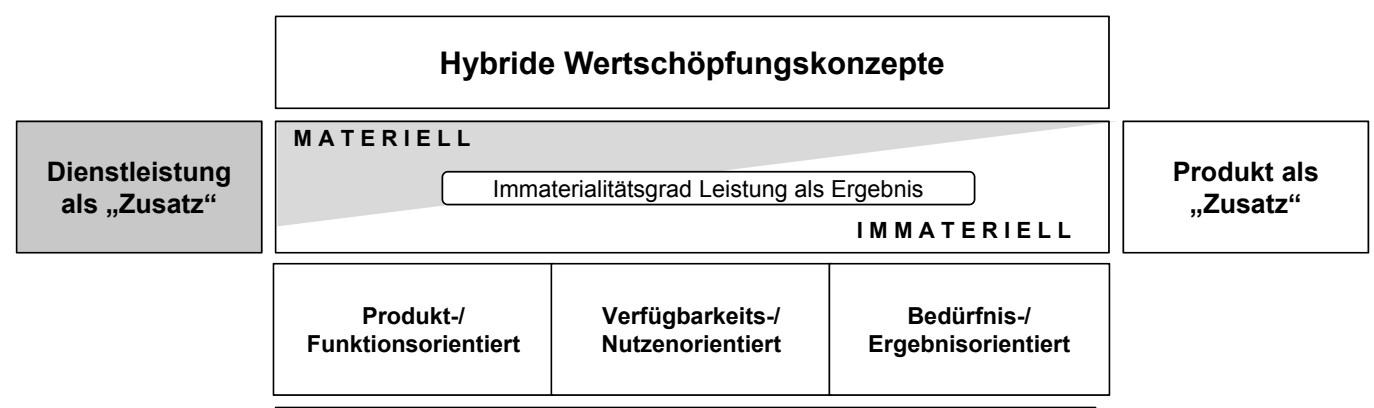

Grad der Integration

Abbildung 2: Einteilung von Produkt Service Systemen (Tukker 2004)

Für die skizzierten Bereiche gelten dabei die gleichen Randbedingungen des Marktes für Industrieprodukte, die sich durch eine Reihe von Tendenzen charakterisieren lassen. Zum einen führt in vielen Fällen eine steigende Produktkomplexität zu einer erhöhten Erklärungsbedürftigkeit vieler Produkte. Zum anderen ist der klassische heimische Markt für viele Anbieter oft bereits gesättigt, sodass Wachstum nur über Exporte möglich erscheint. Das zusätzliche Aufkommen von Wettbewerbern aus Schwellenländern mit einhergehender Abnahme des technischen Vorsprungs gegenüber diesen erfordert zudem zusätzliche und innovative Differenzierungsmöglichkeiten, da ein Wettbewerb über den Preis nicht erfolgsversprechend erscheint (Lay/Rainfurth 2001). Folglich werden industrielle Dienstleistungen immer wichtiger als Differenzierungsmerkmal für Produzenten materieller Güter und können zu Wettbewerbsvorteilen für diese Anbieter führen (Voeth/Bertels 2014). Durch die skizzierte Entwicklung des Marktes für Industrieprodukte wird davon ausgegangen, dass sich viele Produkthersteller gegenwärtig auf einem Transformationspfad befinden. Eine kontinuierliche Entwicklung über die Zeit vom reinen Produzenten eines materiellen Gutes über das strategische Angebot verfügbarkeitsorientierter Dienstleistungen bis hin zum innovativen produzierenden Dienstleistungsanbieter scheint theoretisch zumindest möglich (Oliva/Kallenberg 2003; Gebauer 2004; Gebauer et al. 2005; Penttinen/ Palmer 2007; Gebauer et al. 2008; Matthyssens/Vandenbempt 2008, 2010).

Eine modellhafte Wandlung vom Produzenten zum produzierenden Dienstleistungsanbieter findet sich z.B. bei Meier (2004) sowie bei Spath und Demuß (2003). Ein idealtypisches Stufenmodell der "Servitization" beginnt demnach bei klassischen industriellen Dienstleistungen, die nur als Zusatz zur eigentlichen Leistung gesehen werden. In einem ersten Entwicklungsschritt werden dann zusätzliche Services wie z.B. Installationsarbeiten angeboten, bevor Wartung und Reparaturdienste dazu kommen. Darauf aufbauend lassen sich dann produktfernere Dienstleistungen wie z.B. Finanzierung und Leasing hinzuziehen. Neben dem Angebot von Verfügbarkeitsgarantien endet die letzte Stufe der stärksten Dienstleistungsorientierung schließlich in der Übernahme von direkter Produktionsverantwortung, z.B. in der Form von Betreibermodellen. Der Vorteil solcher Produkt Service Sys- 
teme kann dann anhand des so genannten Service Value gemessen werden (Buschak et al. 2014).

In diesem Zusammenhang erscheint es wichtig, auch die Erfolgsfaktoren dieser zunehmenden Dienstleistungsorientierung zu identifizieren. Die ursprüngliche Annahme war dabei, dass Tertiarisierung ausschließlich Vorteile für Wirtschaft und Gesellschaft schafft (Lay 2014). Mittlerweile werden jedoch nicht nur die unbestreitbaren Vorteile, sondern auch die Herausforderungen der Dienstleistungsorientierung stärker betrachtet. Beispielsweise analysieren Oliva und Kallenberg (2003) die Aufgaben, die Produzenten beim Übergang vom Produkthersteller zum Dienstleister meistern müssen. Ihre Ergebnisse zeigen, dass die Änderung hin zu einer stärkeren Dienstleistungsorientierung eine spezifische Unternehmenskultur, neue Organisationsstrukturen und Prozesse sowie Personal mit ausreichenden Qualifikationen erfordert. Das Fehlen von organisationalen Änderungen für die Bereitstellung von Dienstleistungen sowie die Zurückhaltung von Führungskräften in Unternehmen, die ihr Service-Geschäft erweitern wollen, kann im so genannten „Service Paradox" resultieren. Dieses Phänomen, das durch Gebaner et al. (2005) geprägt wurde, beschreibt die Situation, in der eine Investition, die getätigt wurde, um das Dienstleistungsangebot zu erweitern, zwar zu einem erhöhten Service-Angebot führt, aber gleichzeitig auch zu höheren Kosten und damit paradoxerweise nicht zu entsprechend höheren Erträgen für das anbietende Unternehmen.

Daher müssen für produzierende Unternehmen spezifische Dienstleistungsstrategien entworfen werden (Gebauer et al. 2008; Baines et al. 2009). Dabei kann zwischen Dienstleistungen, die insbesondere das Produkt unterstützen und Dienstleistungen, die in erster Linie den Kunden unterstützen, unterschieden werden (More 2001; Penttinen/Palmer 2007). Erfahrungen zeigen, dass Produzenten erst nachdem sie erfolgreich produktunterstützende Dienstleistungen anbieten, sich zusätzlich auch auf kundenunterstützende Dienstleistungen konzentrieren sollten. Zudem kann die Umstellung nicht umgehend erfolgen, sondern sollte als ein mittel- bis langfristiger Prozess angesehen werden (Velamuri et al. 2011).

\subsection{Digitalisierung industrieller Dienstleistungen}

Nicht nur die Tertiarisierung, sondern in immer stärkerem Ausmaße auch der Trend der Digitalisierung beeinflussen zunehmend das Angebot industrieller Dienstleistungen. Viele Produkthersteller beginnen verstärkt, digitale Systeme für ihre Dienstleistungsangebote einzusetzen. So wird seit einigen Jahren die digitale Unterstützung zur Erbringung von Dienstleistungen unter dem Begriff des Teleservice zusammengefasst (Borgmeier 2002). Dienstleistungen aus dem Bereich des Teleservice verfolgen dabei das Ziel, mit Hilfe digitaler Systeme aus der Ferne Dienstleistungen an Produkten beim Kunden durchführen zu können (Borgmeier 2002).

Teleservices sind durch innovative IKT-Lösungen erst möglich geworden und sind auch auf industriellen Märkten bereits umgesetzt. Jedoch sind ihre Nutzungspotenziale und Anwendungsmöglichkeiten bei weitem noch nicht ausgeschöpft (Schniering 2009). Durch die IKT-Unterstützung können in erster Linie traditionelle produktbegleitende Dienstleistungen weiterentwickelt und derart neue Möglichkeiten im Potenzial- und Prozessbereich des Services ermöglicht werden (Schniering 2009). Jedoch erscheint es erforderlich, die Teleservices stets in ein umfassendes Dienstleistungskonzept einzubinden, um dem Kunden auch den zusätzlichen Nutzen besser kommunizieren zu können (Kirsch 2004). 
In den vergangenen Jahren sind durch die zunehmende Verbreitung von Breitbandinternet insbesondere internetbasierte Dienstleistungen in den Blickpunkt gerückt (Münster/ Meiren 2011). Insbesondere für das Ersatzteilgeschäft und die technische Dokumentation ergeben sich durch diese internetbasierten Dienstleistungen neue Möglichkeiten. Die zunehmende Nutzung des Internets wird vor allem dann als sinnvoll erachtet, wenn das bereits bestehende Dienstleistungsgeschäft ergänzt und optimiert werden soll bzw. um neue technologische Möglichkeiten bestmöglich auszunutzen. Produzenten, die derartige Dienstleistungen anbieten, wollen damit den Kundennutzen steigern und die Kundenbindung erhöhen. Ebenfalls versprechen sich die Anbieter internetbasierter Dienstleistungen eine positive Beeinflussung des Unternehmensimages sowie eine Wirtschaftlichkeitssteigerung (Münster/Meiren 2011).

Der zunehmende Einsatz von Informationstechnik bei produktbegleitenden Dienstleistungen beschränkt sich aber nicht nur auf die Integration des Internet in den Vertrieb und in den Prozess der Leistungserstellung und -erbringung, sondern kann auch durch den Einsatz von Virtual Reality z.B. im Entwicklungsprozess neuer Dienstleistungen zum Ausdruck kommen. Zunehmend beginnen Produzenten damit, ihre Produkte mit intelligenten Systemen auszustatten, die eine Vernetzung von Maschinen und Anlagen in der Produktion ermöglichen. Entwicklungen in dieser Hinsicht werden oft unter dem Überbegriff Industrie 4.0 zusammengefasst (Spath et al. 2013; Baner et al. 2014).

Aufbauend auf den obigen Ausführungen lässt sich der Beitrag digitaler Komponenten für das Dienstleistungsgeschäft in drei aufeinander aufbauende Stufen einteilen. Stehen ganz zu Anfang eher IT-Standardlösungen ohne spezifische Anpassungen im Vordergrund, können in einem zweiten Schritt neue IKT-Lösungen auch zur Verbesserung und Erweiterung der Dienstleistungsangebote eingesetzt werden. Die Verwendung umfassender digitaler Systeme als neuartige Leistungskomponente wäre dann die am stärksten ambitionierte Verwendung einer digitalen Komponente für das Dienstleistungsgeschäft eines produzierenden Unternehmens.

Die beiden aufgezeigten Trends sind zwar beide sowohl in der Literatur ausführlich dokumentiert und zudem durchaus auch in der Praxis zu beobachten, jedoch bleibt weiterhin offen, wie sich die Zusammenhänge zwischen ihnen darstellen bzw. inwieweit sie sich gegenseitig beeinflussen. Neben den bereits verbreiteten Formen der Digitalisierung industrieller Dienstleistungen, wie z.B. dem klassischen Teleservice, werden in Zukunft neue innovative Dienstleistungen und kombinierte Produkt Service Systeme möglich. Daher soll im Folgenden die Auswirkung der Digitalisierung auf industrielle Dienstleistungsangebote anhand von drei voneinander unabhängigen Beispielen detaillierter untersucht werden.

\section{Auswirkungen der Digitalisierung auf industrielle Dienstleistungen}

\subsection{Formen der Digitalisierung von Dienstleistungsangeboten bei Produktherstellern}

Die im Folgenden aufgeführten Formen der Digitalisierung bzgl. des Angebots industrieller Dienstleistungen stammen aus Fallstudien, die auf einer Zusammenarbeit der Autoren mit produzierenden Unternehmen gründen. Diese wurden in den vergangenen Jahren, von 2008 bis 2012, im Rahmen verschiedener Forschungsprojekte durchgeführt und erarbeitet. Die vorliegenden und hier aufgeführten Informationen stammen aus verschiedenen Datenquellen. Die Hauptdatenquelle basiert auf Gesprächsprotokollen, die im Rahmen der Forschungsprojekte der Autoren erstellt wurden. Weitere Datenquellen stammen z.B. 
aus Publikationen in Fachzeitschriften und dem Internetauftritt der drei Unternehmen. Für den vorliegenden Beitrag wurden die Anwendungsfälle neu analysiert und ausgewertet und insbesondere hinsichtlich ihres Beitrags zur Digitalisierung untersucht. Alle drei unten beschriebenen Unternehmen stammen aus dem Maschinen- und Anlagenbau und weisen vergleichsweise ausgeprägte Serviceangebote auf, die in jeweils unterschiedlicher Weise mit der Digitalisierung bzw. dem Einsatz von IKT-Lösungen in Zusammenhang stehen. Für den Beitrag wurden genau diese Unternehmen gewählt, da diese die Digitalisierung für jeweils unterschiedliche Zwecke und Ziele bezüglich ihres Dienstleistungsangebots einsetzen.

\section{Digitalisierung I - Einsatz digitaler Systeme für produktbegleitende Dienstleistungen}

Die erste Form der Digitalisierung lässt sich anhand des Beispiels eines Lackieranlagenherstellers, dessen Hauptsitz in Süddeutschland liegt, erläutern. Das hier betrachtete Unternehmen erzielt etwa einen jährlichen Umsatz von 400 Mio. EUR weltweit und beschäftigt circa 1.700 Mitarbeiter, wovon 350 in Deutschland tätig sind. Der Hersteller von Lackieranlagen stellt einerseits Produkte für Endkunden her, andererseits werden für die Industrie Lösungen für Pulverbeschichtungen und Flüssiglacke entwickelt und gefertigt.

Das Servicegeschäft des Lackieranlagenherstellers konnte in den letzten Jahren insbesondere für industrielle Anwendungen kontinuierlich ausgebaut werden. So existieren neben der Inbetriebnahme auch Wartungen und Reparaturen, eine Service-Hotline sowie verschiedene Angebote aus dem Bereich der Engineering Dienstleistungen und der Projektplanung. Besonders hervorzuheben ist das Schulungsangebot, das modular aufgebaut ist und dessen Inhalte in dieser Form nicht bei der Konkurrenz vorzufinden sind und bei der Einführung im Jahr 2008 in gewisser Weise eine Marktinnovation darstellte.

Da das Unternehmen global agiert und ein Großteil der Kunden in Europa und in Asien angesiedelt ist, weist der Hersteller eine relativ hohe Exportquote von circa 50 Prozent im Produktgeschäft auf. Dieser hohe Exportanteil stellt das Dienstleistungsgeschäft allerdings vor die Herausforderung, die angebotenen Services auch über weite Distanzen zu exportieren. Da bei der Internationalisierung von Dienstleistungen grundsätzlich die Schwierigkeit des gleichzeitigen Anfalls von Produktion und Konsum auftritt (Erramilli 1989; Vandermerwe/Chadwick 1989), ist der Hersteller dazu übergegangen, digitale Systeme für die Abwicklung seiner Dienstleistungen im Ausland einzuführen. Hierdurch gelingt eine gewisse Entkopplung von Produktion und Konsumtion, wodurch das Uno-actu-Prinzip teilweise außer Kraft gesetzt werden kann (Knight 1999).

Für die praktische Umsetzung hat das Unternehmen hierzu so genannte Service-Videos entwickelt, die für den Bereich der Wartung, Reparatur und Schulung eingesetzt werden. Die Service-Videos werden dem Kunden direkt mit der Anlage auf DVD mitgeliefert. In den Videos wurde weitgehend auf Sprache verzichtet, sondern so weit wie möglich mit Piktogrammen gearbeitet. Auf den Videos sind kurze Filmsequenzen abgelegt, die eine bestimmte Anwendung vorführen und auf Basis von Gesten z.B. das Zerlegen von Bauteilgruppen beschreiben und wie defekte Teile gewechselt werden können. Diese Filmsequenzen beinhalten Schulungselemente, sind aber auch für die Wartung und Reparatur von Anlagen einsetzbar. Hierdurch können einfachere Instandhaltungsmaßnahmen und die Grundlagen einer Anlagenanwendung ohne Präsenz von Servicetechnikern aus Deutschland vermittelt bzw. durchgeführt werden. 
Bei der Einführung der Service-Videos im Jahr 2009 stellte diese neuartige Lösung eine grundlegende Verbesserung des Servicegeschäfts mit weitreichenden Vorteilen für Anbieter und Kunde dar. So konnten nicht nur anfallende Reise- und Arbeitskosten deutlich reduziert, sondern auch vor Ort beim Kunden sehr viel schneller reagiert werden, wodurch sich Ausfallzeiten deutlich reduzieren ließen. Mit Hilfe der Service-Videos war der Hersteller fortan in der Lage, wertvolle Ressourcen, in Form von Servicetechnikern, deutlich effizienter einzusetzen. Der Kunde konnte hingegen ohne zeitlichen Verzug einfachere Arbeiten an der Lackieranlage selbst durchführen, wodurch geringere Ausfallzeiten bzw. höhere Verfügbarkeiten entstehen können.

Wie das Beispiel zeigt, konnten weite Teile des Servicegeschäfts des Herstellers mit Hilfe relativ einfacher IT-Standardlösungen grundlegend verbessert werden. Zwar wurden diese Dienstleistungen schon vor dem Einsatz der Service-Videos angeboten bzw. könnten grundsätzlich auch klassisch und ohne diese digitale Komponente durchgeführt werden. Dennoch war es möglich, nicht nur die Abwicklung der Dienstleistung zu beschleunigen, sondern auch die Qualität des Angebots für den Kunden zu erhöhen. Folglich konnten die anfallenden Kosten von Serviceeinsätzen, bei gleichzeitiger Erhöhung der Qualität, für einen Großteil des Dienstleistungsgeschäfts reduziert werden. Der Einsatz digitaler Systeme zur Verbesserung von Dienstleistungen kann daher als Grundstufe der Digitalisierung angesehen werden.

Weitere Beispiele zu dieser Digitalisierungsform werden auch in der Literatur beschrieben und beschränken sich bei weitem nicht nur auf Service-Videos oder auf die Entkopplung von Produktion und Konsumtion. Ein Beispiel, das häufig in der Literatur genannt wird, fällt unter den Sammelbegriff des Teleservice. Diese Anwendungen verfolgen das Ziel, Dienstleistungen aus der Ferne mit Hilfe digitaler Systeme an Produkten durchführen zu können, ohne sich dabei auf eine bestimmte Dienstleistung zu beziehen (Borgmeier 2002). Hierzu kann auch der Einsatz von Internet, von mobilen Endgeräten oder Virtual Reality bzw. Augmented Reality Anwendungen zählen (Münster/Meiren 2011), welche Dienstleistungen verbessern oder entscheidend unterstützen.

\section{Digitalisierung II - Reine digitale Dienstleistungen}

Eine weitere Form der Digitalisierung bei Dienstleistungsangeboten von Produzenten geht über den Einsatz digitaler Systeme zur Dienstleistungsverbesserung hinaus. Hierzu kann der Fall eines kleinen Maschinenbauunternehmens mit Sitz in Baden-Württemberg herangezogen werden. Der Maschinenbauer beschäftigt etwa 50 Mitarbeiter und generiert damit einen jährlichen Gesamtumsatz von etwa 3,2 Mio. EUR. Der Betrieb stellt Maschinenkomponenten her, die in Anlagen verbaut werden, die dann wiederum als Teil von Fertigungsstraßen integriert sind. Die Maschinenkomponenten werden für Kunden aus der Automobilindustrie sowie aus dem Energiesektor gefertigt. Das Unternehmen weist ein ausgeprägtes Dienstleistungsgeschäft auf, das einen Umsatzanteil von etwa 30 Prozent am Gesamtumsatz ausmacht. Hierzu gehören neben Wartung und Reparatur auch eine ServiceHotline sowie diverse Engineering Dienstleistungen. Insbesondere bei diesen Engineering Dienstleistungen konnte der Maschinenbauer in den letzen Jahren neuartige Angebote generieren, die größtenteils durch den gezielten Einsatz von Informations- und Kommunikationstechnologien zu Stande kamen.

Hier kann als erstes Beispiel das Angebot von Frequenzanalysen genannt werden. Mit Hilfe von Sensortechniken und Steuerungselementen, die in die Maschinenkomponenten 
vor der Auslieferung an den Kunden eingebaut werden, ist es möglich, Analysen zu Zustandsveränderungen an Bauteilen, auf Basis von Schwingungen und akustischen Signalen, durchzuführen, die dann mit Hilfe eines Rechners ausgewertet werden. Hieraus lassen sich z.B. Fehlerquellen bei Ausfällen identifizieren, Brüche in den Komponenten feststellen oder sonstige Veränderungen der mechanischen Strukturen erkennen, woraus sich wiederum Rückschlüsse auf Belastungen und Temperaturentwicklungen ziehen lassen. Das Beispiel des Angebots der Frequenzanalyse basiert folglich rein auf digitalen Elementen.

Als zweites Beispiel lässt sich das Erstellen von Werkzeugplänen heranziehen, welche auf Basis von CAD-Spezialsoftware entwickelt werden und deren vollständige Funktionalität nur in digitaler Form genutzt werden können. Durch diese Anwendung ergeben sich weitreichende Vorteile im Bereich der Konstruktion und der Auslegung der Maschinenkomponenten. Da die Pläne digital verankert sind, lassen sich auch Tests und Simulationen, z.B. zur Belastbarkeit und Funktionsfähigkeit der einzelnen Teile der Maschinenkomponenten, durchführen. Auch diese Dienstleistung ist somit nur durch den Einsatz digitaler Systeme, hier in Form von CAD-Spezialsoftware, möglich.

Wie der Fall dieser Engineering Services zeigt, weisen die vorliegenden Dienstleistungen den Charakter eines digitalen Leistungsergebnisses auf und sind somit ohne digitale Komponente nicht durchführbar bzw. auch nicht existenzfähig. Durch den stark digitalen Charakter, insbesondere auf der Dimension des Leistungsergebnisses, stellen diese Dienstleistungen eine grundsätzliche Erweiterung des Angebotsspektrums dar, auch da deren Existenz zuvor, ohne IT-Einsatz, nicht möglich war. Demnach ergibt sich ein grundlegender Unterschied zur ersten Form der Digitalisierung: Beim Einsatz von digitalen Systemen zur Verbesserung produktbegleitender Dienstleistungen kommt es folglich zu Veränderungen auf der Potenzial- und Prozessebene im Leistungserstellungsprozess (vgl. auch Schniering 2009). Im Gegensatz dazu nehmen reine digitale Dienstleistungen auch eine Änderung auf der Ergebnisebene vor, i.S. eines immateriell-digitalen Leistungsergebnisses. Folglich leitet sich hieraus eine Nichtexistenzfähigkeit dieser Dienstleistungen ohne digitale Systeme ab.

Diese Dienstleistungen erweitern folglich das Angebotsspektrum eines Herstellers gegenüber traditionellen Services, weisen einen stark digitalen Charakter auf und sind ohne digitale Komponente nicht durchführbar, wodurch deren Leistungsergebnis ohne Digitalisierung nicht wirksam werden könnte. Aufgrund dieser Eigenschaften wird diese Digitalisierungsform als reine digitale Dienstleistung bezeichnet. Diese Dienstleistungen beschränken sich jedoch nicht nur auf Engineering Dienstleistungen. In der Literatur werden hierzu z.B. auch die Service-Hotline und Online-Support-Systeme wie User Helpdesks gezählt (vgl. hierzu Münster/Meiren 2011).

\section{Digitalisierung III - Digitale Produkt Service Systeme}

Für eine dritte Form der Digitalisierung von Dienstleistungen kann der Anwendungsfall eines Herstellers von Fertigungslösungen betrachtet werden. Das weltweit agierende Unternehmen mit mehreren Standorten in Deutschland und Hauptsitz in Baden-Württemberg stellt Werkzeugmaschinen her, beschäftigt in Deutschland etwa 1.700 Mitarbeiter und erwirtschaftet einen jährlichen Umsatz von etwa 320 Mio. EUR. Der Werkzeugmaschinenhersteller beliefert Unternehmen aus der Automobilindustrie und weist ein umfangreiches Dienstleistungsangebot auf, welches über diverse Servicestützpunkte in Europa, Amerika und Asien vertrieben und angeboten wird. Zum Angebotsspektrum zählen z.B. technischer Support, Wartungen und Reparatureinsätze, Ersatzteilservice, Training von Fachkräften, 
Inspektionen, Online Helpdesk sowie Modernisierung und Retrofitting von Gebrauchtmaschinen.

Im vorliegenden Fall verlangte ein zentraler Kunde Angaben zu Verfügbarkeiten für verschiedene Bauteile und Bauteilgruppen vom Werkzeugmaschinenhersteller. Basierend auf diesen Verfügbarkeiten sollten für die Zukunft dann Lebenszykluskostengarantien für die verschiedene Komponenten definiert werden. Diese sollten über verschiedene Kennzahlen wie die Mean Time between Failure (MTBF), die Mean Time to Repair (MTTR) und die Mean Costs for Replacement Parts (MCRP) vertraglich fixiert werden. Hierunter werden dann die kompletten Kosten für die Instandhaltung der Anlage über die nächsten Jahre gefasst und vertraglich geregelt. Das Ziel des Kunden aus der Automobilwirtschaft war dabei, zukünftig Verfügbarkeits- und Lebenszykluskostengarantien vertraglich festzulegen und so die gesamten Lebenszykluskosten der Anlage transparent und steuerbar zu machen. Bei Abschluss eines solchen Vertrags zwischen Werkzeugmaschinenhersteller und Automobilhersteller sind dann zwei Szenarien denkbar: wird der vertraglich festgelegte Wert für die verschiedenen Kennzahlen erreicht, so gilt die Lebenszykluskostengarantie als erfüllt. Liegt der Wert der Bauteilkennzahlen jedoch unter dem vertraglich definierten Wert, so hätte sich der Hersteller an den Folgekosten zu beteiligen.

Der Werkzeugmaschinenhersteller wurde bezüglich der Abgabe von Verfügbarkeiten und Lebenszykluskosten vor große Herausforderungen gestellt, da keinerlei Erfahrungen zu den verlangten Kennzahlen aus der Vergangenheit vorlagen. Allerdings schien eine erfolgreiche Identifikation der Kennzahlen äußerst erfolgsversprechend, da mit der Abgabe vertraglich garantierter Verfügbarkeits- und Kostengarantien sich weitreichende Wettbewerbsvorteile für den Hersteller ergeben könnten. Daher wurde auf Basis der in den Werkzeugmaschinen enthaltenen Condition Monitoring Systemen, die bereits in den Anlagen der Kunden installiert waren, im Nachhinein die vorliegenden Dokumentationen zu Ausfallverhalten, Fehlerursachen und Ersatzteilbedarfen ausgewertet und hinsichtlich einer vergangenheitsbasierten Verfügbarkeit der einzelnen Bauteile analysiert. Hierdurch war es schließlich möglich, konkrete Werte zu MTBF, MTTR und MCRP gezielt für einzelne Bauteile und sogar für ganze Bauteilgruppen zu ermitteln. Mit Hilfe dieses Vorgehens war eine erste Grundlage geschaffen, bei Bedarf gezielt vertraglich zugesicherte Angaben zu Verfügbarkeiten und Lebenszykluskostengarantien gegenüber Kunden abgeben zu können.

Dennoch war dies nicht das letzte Ziel des Maschinenherstellers, mit diesen Verträgen umzugehen. Vielmehr war es die Vision des Werkzeugmaschinenherstellers, ein verfügbarkeits- und kostenorientiertes Produkt Service System zu schaffen, welches mit Hilfe einer digitalen Komponente, dem Condition Monitoring System, jederzeit überwacht und gesteuert werden konnte und somit systematisch die vereinbarte Leistungsgarantie gegenüber dem Kunden erbringen konnte. Durch diese Überwachungs- und Steuerungsfunktion war der Hersteller in der Lage, das mit Produkt Service Systemen einhergehende Risiko deutlich zu reduzieren und somit einen entscheidenden Schritt zum Dienstleistungsanbieter zu machen.

Wie der Fall des Werkzeugmaschinenherstellers zeigt, wurde ein digitales System gezielt in ein nutzungsorientiertes Produkt Service System integriert, um dies durch Überwachung und Steuerung transparenter für den Hersteller zu machen. Neben einer materiellen und immateriellen Leistungskomponente besteht dieses Produkt Service System somit auch aus einer digitalen Komponente, welcher ein relevanter Beitrag für das erfolgreiche Angebot dieser Verfügbarkeits- und Kostengarantien zukommt. Solch ein digitales Produkt Service 
System zielt somit auf ein Leistungsangebot für den Kunden ab, das mit Hilfe einer digitalen Komponente umgesetzt und realisiert wird.

In dieser Form der Digitalisierung versuchen produzierende Unternehmen digitale Systeme so einzusetzen, dass diese als dritte Leistungskomponente im Rahmen des Angebots von Produkt Service Systemen fungieren. Mit Hilfe dieser digitalen Leistungskomponente können folglich Produkt Service Systeme nicht nur verbessert, sondern wie im Fall des Werkzeugmaschinenherstellers möglicherweise überhaupt erst angeboten werden. Dem integrativen Einsatz von Informations- und Kommunikationstechnologien (IKT) kommt bei Produkt Service Systemen somit eine zentrale Rolle zu, der die materiellen und immateriellen Bestandteile des Leistungsbündels entscheidend erweitert.

\subsection{Wechselwirkungen von Digitalisierung und Tertiarisierung auf betrieblicher Ebene}

Nach den oben aufgeführten Beispielen stellt sich die Frage, inwieweit der Trend der Digitalisierung und der Trend der Tertiarisierung gegenseitig in Wechselwirkungen stehen. Zwar kann davon ausgegangen werden, dass beide Trends aus der Veränderung externer Rahmenbedingungen resultieren. Allerdings scheinen hier Unterschiede hinsichtlich Ursache und Wirkung beim Einsatz digitaler Systeme in Dienstleistungsangeboten zu existieren.

Die Literatur geht davon aus, dass der Trend der Tertiarisierung insbesondere durch sich verändernde Markt- und Nachfragebedingungen in der Industrie ausgelöst wird (Gebauer 2004). Hier werden z.B. sich verkürzende Lebenszyklen der Produkte bei gleichzeitiger Erhöhung der Produktvarianten (Schröter et al. 2008) oder geringe Differenzierungsmöglichkeiten durch die Homogenisierung des Produktgeschäfts mit einhergehenden schrumpfenden Margen (Belz et al. 1997) genannt. Hieraus ergibt sich für Produzenten die Notwendigkeit, sich vom reinen Produzenten zum Dienstleistungsanbieter zu wandeln, um die Wettbewerbsfähigkeit zu erhalten oder sogar auszubauen (Gebauer 2004). Die Digitalisierung traditioneller Industrien ist hingegen auf Fortschritte in der Technologieentwicklung zurückzuführen. Durch schnelle Verbesserungen bei IKT wird es für Produkthersteller möglich, ihre Produkte mit intelligenten Komponenten zu versehen (Spath et al. 2013; Baner et al. 2014). Dieser Trend zeichnet sich durch die Entwicklungen von traditionellen Industrien hin zu einer Industrie $4.0 \mathrm{ab}$. In der Industrie kommt es somit zwangsläufig zu einer Verschmelzung beider Trends.

Daraus zeigt sich, dass sowohl die Digitalisierung als auch die Tertiarisierung als Entwicklungen zu verstehen sind, die für traditionelle Produkthersteller neue Aspekte jenseits des Kerngeschäfts darstellen. Durch die Verschmelzung beider Trends, im Sinne einer Digitalisierung von Dienstleistungen, ergeben sich für Produkthersteller nochmals deutlich erhöhte Anforderungen. Die Wechselwirkungen aus Digitalisierung und Tertiarisierung beschreiben somit einen neuen, aus zwei Trends bestehenden Transformationspfad, auf dem sich Produkthersteller befinden. Hieraus ergeben sich neue Chancen, aber auch Risiken für Produkthersteller.

Wie die erste Form der Digitalisierung im Falle des Lackieranlagenherstellers zeigt, wurde der Einsatz von Servicevideos insbesondere deswegen vorangetrieben, weil das Unternehmen begann, das eigene Servicegeschäft auszubauen und in Länder mit hoher räumlicher und kultureller Distanz zu exportieren. Der Einsatz digitaler Trägermedien, wie im Falle der Servicevideos, stellte in diesem Fall eine hervorragende Möglichkeit einer Entkopplung von Dienstleistungserbringung und Dienstleistungskonsum dar und somit die Aussetzung des Uno-actu-Prinzips (vgl. hierzu z.B. Beckenbauer 2006; Wassermann 
2010). In diesem Fall ist davon auszugehen, dass die zusätzliche Dienstleistungsorientierung des Lackieranlagenherstellers einen Einsatz von IKT-Systemen bedingte, also eine Weiterentwicklung auf dem Weg zum Dienstleistungsanbieter den Grad der Digitalisierung erhöhte. Im Gegenzug wurde allerdings das Geschäft des Dienstleistungsexports überhaupt erst in diesem Umfang durch den Einsatz digitaler Trägermedien möglich. Folglich bedingte die Möglichkeit einer höheren Digitalisierungsform auch erst den verstärkten Ausbau des Dienstleistungsgeschäfts. Demnach ist für den Fall des Lackieranlagenherstellers von Wechselwirkungen zwischen Dienstleistungsorientierung und Digitalisierungsgrad auszugehen, durch welche sich die beiden Trends gegenseitig beschleunigen.

Ebenfalls existieren Wechselwirkungen im Fall des Maschinenkomponentenherstellers. Wie oben bereits erläutert, wurde die Dienstleistung der Frequenzanalyse überhaupt nur möglich, weil neue technologische Möglichkeiten geschaffen wurden, um solche Schwingungsanalysen und akustischen Signale durchzuführen. In diesem Fall ist das Leistungsergebnis der Dienstleistung selbst digital (Münster/Meiren 2011) und wäre durch andere traditionelle Techniken nicht durchführ- oder realisierbar und somit nicht existenzfähig. In diesem Fall, also bei Dienstleistungen, deren Leistungsergebnis nicht mehr nur immateriell, sondern auch einen digitalen Charakter aufweist, kann davon ausgegangen werden, dass per se Wechselwirkungen auftreten. So sind in der Literatur beschriebene digitale Anwendungen, wie Tests und Simulationen, Kunden-Apps, Online-Dienste oder sogar Augmented- und Virtual Reality-Anwendungen als eine Art der Digitalisierungsform zu verstehen, die eine Beschleunigung der Transformation von Produzenten zu Dienstleistungsanbietern bewirken.

Die dritte Digitalisierungsform, die durch das Beispiel des Werkzeugmaschinenherstellers verdeutlicht wurde, versteht hingegen die Digitalisierung als dritte Leistungskomponente im Rahmen eines Leistungsbündels aus materiellen und immateriellen Bestandteilen. Demnach erfolgt hier eine Verschiebung weg von hybriden Leistungsbündeln hin zu Leistungsbündeln bestehend aus drei Leistungskomponenten. Diese digitalen Produkt-Service Systeme $\left(\mathrm{dPS}^{2}\right)$ greifen folglich auf innovative Kombinationen aus digitalen, materiellen und immateriellen Komponenten zurück, um neuartige Leistungsbündel anzubieten und hierdurch neue Wettbewerbspotenziale erschließen zu können. Durch die Nutzung einer dritten Leistungskomponente kommt es auch hier zu gegenseitigen Wechselwirkungen. So wird einerseits erst durch den Einsatz intelligenter Systeme das Angebot von Verfügbarkeitsgarantien möglich, wie im Falle des Werkzeugmaschinenherstellers. Nur mit Hilfe eines verstärkten Zugangs zu Informationen über die Betriebseigenschaften des eigenen Produkts sowie einer höheren Kontrolle des Produzenten über den Betriebszustand der Anlagen können die Risiken für den Anbieter soweit abgebaut werden, dass eine Verfügbarkeitsgarantie unter wirtschaftlich rentablen Bedingungen angeboten werden kann. In diesem Fall ist also die Digitalsierung als Treiber für eine erhöhte Dienstleistungsorientierung zu sehen.

Andererseits ist aber auch der Fall denkbar, dass Produzenten, die solche Produkt Service Systeme einführen möchten, nun die Möglichkeit haben, mit Hilfe einer zusätzlichen digitalen Komponente leistungsbezogene Angebote gegenüber ihren Kunden offerieren zu können. In diesem Fall wäre also der Ausbau des Dienstleistungsgeschäfts der Treiber für einen höheren Digitalisierungsgrad des Unternehmens.

In Anbetracht der drei hier vorgestellten Digitalisierungsformen kann davon ausgegangen werden, dass die beiden Trends in der Industrie gegenseitig in Wechselwirkung stehen 
und sich somit gegenseitig beschleunigen. So kann einerseits der Ausbau des Dienstleistungsgeschäfts von Produzenten dazu führen, dass der Einsatz von IKT-Systemen notwendig wird, um ein attraktives und wirtschaftliches Dienstleistungsangebot entwickeln und erbringen zu können. Andererseits ergibt sich aber auch die Möglichkeit, dass Produzenten mit einer höheren IKT-Orientierung in der Lage sind, ihr Dienstleistungsangebot verstärkt auszubauen und somit höhere Entwicklungsstufen des Transformationspfads zu erreichen.

Somit hätte nicht nur das Dienstleistungsangebot eines Produzenten systemischen Charakter (Meyer/Blümelhuber 1998), sondern so würde auch der Umfang des Einsatzes von Informations- und Kommunikationstechnologien systemische Eigenschaften im Dienstleistungsgeschäft aufweisen. Infolgedessen sollten auch hier in Zukunft Muster aus Leistungsbündeln mit materiellen, immateriellen und digitalen Komponenten in der Praxis vorzufinden sein. Entsprechend der hier beschriebenen Digitalisierungsformen lässt sich nun ein Transformationspfad entwickeln, der die beiden Trends und ihre Wechselwirkungen berücksichtigt.

\section{Ein erweitertes Transformationsmodell unter dem Einfluss der Digitalisierung}

Wie zu Beginn des Beitrags bereits vorgestellt, existieren in der Literatur Modelle, welche die Transformation von Produzenten zu Dienstleistungsanbietern beschreiben. In diesen Modellen fehlt bislang aber eine Berücksichtigung einer zunehmenden Digitalisierung der Industrie. Folglich ergeben sich Erweiterungsoptionen für solche Transformationsmodelle, welche beide Trends berücksichtigen. Zur Beschreibung von Entwicklungspotenzialen entlang der Transformation lassen sich die oben diskutierten Digitalisierungsformen heranziehen.

Die drei Formen orientieren sich jedoch weniger an der Komplexität eingesetzter IKTLösungen, sondern vielmehr am Zweck, zu welchem diese im Rahmen der Dienstleistungserbringung eingesetzt werden. Wie die Beispiele zeigen, reichen die Ziele für den Einsatz von IKT bei Serviceangeboten von einer Verbesserung angebotener Dienstleistung über eine Portfolio-Erweiterung des Dienstleistungsangebots bis zur Integration einer digitalen Komponente in ein Leistungsbündel mit materiellen und immateriellen Anteilen. Ein geeignetes Transformationsmodell für Produkthersteller, die sich im Einfluss beider Trends befinden, lässt sich durch folgende Entwicklungsperspektiven beschreiben, die aufeinander aufbauen (vgl. Abbildung 3):

- Produzent mit obligatorischen Dienstleistungen weitgehend ohne digitale Komponenten: In der ersten Stufe konzentrieren sich Produkthersteller auf ihr Kerngeschäft. Dienstleistungen werden kaum oder lediglich als Vertriebsunterstützung für das Produkt angeboten. Durch den eher geringen Stellenwert der Dienstleistungen im Unternehmen werden digitale Systeme nicht oder nur sporadisch eingesetzt.

- Anbieter IT-basierter Dienstleistungen (Digitalisierungsform I): Produkthersteller nutzen Dienstleistungen bereits als Differenzierungsmerkmal und eigenständigen Umsatztreiber. Diese Unternehmen setzen digitale Komponenten ein, um ihre Dienstleistungsangebote kontinuierlich zu verbessern. Services können somit nicht nur effizienter abgewickelt, sondern auch zu einer höheren Qualität angeboten werden. Hierdurch lassen sich Differenzierung und Umsatz weiter ausbauen. 
- Anbieter reiner digitaler Dienstleistungen (Digitalisierungsform II): In dieser Stufe erweitern Produkthersteller ihr Dienstleistungsportfolio gezielt mit reinen digitalen Dienstleistungen, um ein noch leistungsfähigeres und kundenorientiertes Angebot offerieren zu können. Die neuen Dienstleistungen waren zuvor so nicht existenzfähig, bieten weiteres Differenzierungspotenzial und steigern die Kundenzufriedenheit durch eine erhöhte Kundennähe.

- Anbieter digitaler Produkt Service Systeme (Digitalisierungsform III): Diese Unternehmen haben ihre Dienstleistungen zum Kerngeschäft ausgebaut und bieten Kunden Problemlösungen mit Leistungsgarantien an. Um diese Produkt Service Systeme noch effizienter und leistungsfähiger zu machen, werden digitale Systeme als neuartige Leistungskomponente verwendet und integriert neben materiellen und immateriellen Komponenten als Problemlösung angeboten. Diese Unternehmen bieten nun digitale Produkt Service Systeme an und eröffnen sich neuartige Wettbewerbspotenziale.

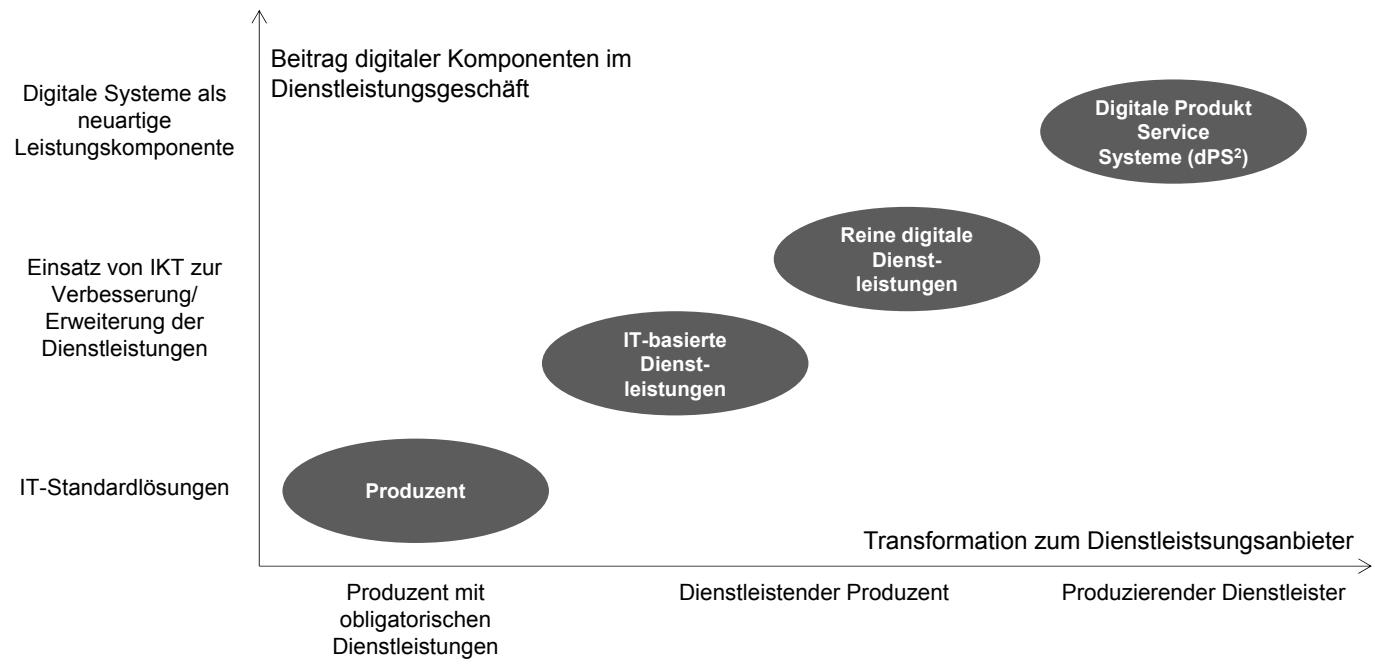

Abbildung 3: Transformationsmodell für Produkthersteller unter dem Einfluss von Tertiarisierung und Digitalisierung

Jenseits dieser Entwicklungsperspektiven ist auch denkbar, dass es Abweichungen vom Transformationspfad bei Unternehmen gibt. So besteht auch die Möglichkeit, dass Betriebe innovative Leistungsbündel traditionell und ohne digitale Komponenten anbieten. In diesem Fall würde sich das Unternehmen stärker vom Trend der Tertiarisierung beeinflussen lassen. Im Gegensatz dazu werden sich andere Betriebe stärker auf die Digitalisierung von Maschinen und Anlagen, also auf das Kernprodukt, konzentrieren. Hier würde sich das Ende des Transformationspfads allerdings durch das Angebot von Cyber-physischen Systemen (Spath et al. 2013), gemäß der Vision einer Industrie 4.0, darstellen. Je nach Einfluss kann sich ein Produzent also mehr oder weniger von der Transformationslinie in eine bestimmte Richtung entfernen.

Gemäß den bereits existierenden Transformationsmodellen ist davon auszugehen, dass die Stufen nacheinander durchlaufen werden, also die Stufen als kontinuierliche Evolution zu sehen sind. Inwieweit in der Praxis Stufen übersprungen werden (können), ist auf Basis 
der drei Fallstudien noch unklar. Im Fall der drei oben beschriebenen Produkthersteller ist von einer kontinuierlichen Entwicklung auszugehen. So beherrscht z.B. das kleine Maschinenbauunternehmen auch die erste Digitalisierungsform, jedoch nicht die Dritte. Der betrachtete Werkzeugmaschinenhersteller wies im Dienstleistungsgeschäft ebenfalls die beiden ersten Digitalisierungsformen auf, bevor die dritte Stufe angestrebt wurde. Der Lackieranlagenhersteller wiederum wendete weder Digitalisierungsformen der zweiten noch der dritten Stufe an, sondern bot ausschließlich IT-basierte Dienstleistungen an.

Durch die oben diskutierten und unterstellten Wechselwirkungen beider Pfade ist davon auszugehen, dass die oben gezeigte Entwicklung eine gewisse Dominanz aufweist und im Zentrum der beiden Trends steht. Demnach wäre die Vermutung, dass Unternehmen, die sich vom dominanten Pfad entfernen, durch die unterstellten Wechselwirkungen, wieder dorthin zurückgetrieben werden. Eine sehr hohe Dienstleistungsorientierung gänzlich ohne digitale Systeme wäre in Zukunft bei weiterem Voranschreiten der Digitalisierung nur schwer vorstellbar. Da obiger Pfad aber nicht zwangsläufig komplett durchlaufen werden muss, wären gewisse Muster in der Praxis zu erwarten, die mehr oder weniger auf den dominanten Entwicklungsperspektiven liegen.

\section{Zusammenfassung}

Der vorliegende Beitrag analysiert die Rolle der Digitalisierung bei einer Transformation vom Produzenten zum Dienstleistungsanbieter. Da hierzu bisher keine Konzepte vorliegen, wurden auf Basis verschiedener Fallstudien diverse Formen der Digitalisierung im Dienstleistungsgeschäft von Produktherstellern aufgezeigt. Hier konnten drei Digitalisierungsformen identifiziert werden: der Einsatz von digitalen Systemen zur Verbesserung produktbegleitender Dienstleistungen (Digitalisierungsform I), reine digitale Dienstleistungen zur Erweiterung des Dienstleistungsportfolios (Digitalisierungsform II) sowie digitale Produkt Service Systeme, welche Digitalisierung als dritte Komponente eines Leistungsbündels integrieren (Digitalisierungsform III).

Hierbei ist zu beachten, dass die Digitalisierungsformen insbesondere den Zweck berücksichtigen, zu welchem digitale Systemen im Dienstleistungsgeschäft eingesetzt werden. Wird diese Zweckgebundenheit auf bestehende Transformationsmodelle übertragen, entstehen vier Entwicklungsstufen, die einen Transformationspfad von Produktherstellern unter Einflüssen der Digitalisierung und der Tertiarisierung in der Industrie über die Zeit beschreiben.

Durch die vorliegenden Fallstudien wurde auch deutlich, dass der Wandel zum Dienstleistungsanbieter in Wechselwirkung zur Digitalsierung steht. So ist davon auszugehen, dass einerseits der Einsatz von IKT die Dienstleistungsorientierung zusätzlich vorantreibt. Andererseits kann festgestellt werden, dass sich bei verstärktem Ausbau des Dienstleistungsgeschäfts der Einsatz digitaler Systeme hinsichtlich Umfang und Intensität erhöht. Daher wird davon ausgegangen, dass die beiden industriellen Trends sich gegenseitig stimulieren und sich beschleunigend auf eine Dienstleistungsorientierung von Produktherstellern auswirken.

Dieses erweiterte Transformationsmodell wurde auf Basis von Fallstudien und einer Einordnung von bestehender Literatur entwickelt. Folglich könnte zukünftig analysiert werden, inwieweit die Entwicklungsstufen in der Praxis realisiert werden bzw. ob sich gewisse Muster ergeben. Dies könnte mit Hilfe statistischer Analysen durchgeführt werden, wodurch sich möglicherweise auch Unterschiede zwischen Branchen identifizieren ließen. 
Ebenfalls wäre es auf Basis von Fallstudien möglich, Hemmnisse und Potenziale entlang des Transformationspfads und den verschiedenen Entwicklungsstufen zu identifizieren, um Handlungsempfehlungen für die Praxis ableiten zu können. Hierzu müssten aber eigens durchgeführte Fallstudien herangezogen werden, die explizit diese Themenstellung bearbeiten.

Somit ist das entwickelte Konzept trotz fehlender Repräsentativität in der Lage, einen Transformationspfad mit Entwicklungsstufen für Produkthersteller unter Einfluss von Digitalisierung und Tertiarisierung aufzuzeigen. Es kann folglich als erstes Rahmenkonzept dienen, welches zwei Trends in der Industrie vereint und ein Verständnis für die Digitalisierung industrieller Dienstleistungen schafft. Eine weitere Ausarbeitung mit Handlungsoptionen sowie Chancen und Risiken für Unternehmen einerseits sowie Analysen zur Tragfähigkeit des Konzepts in der Praxis andererseits bleibt zukünftigen Arbeiten vorbehalten.

\section{Literaturhinweise}

Baines, T./Lightfoot, H./Benedettini, O./Kay, J. (2009): The servitization of manufacturing: A review of literature and reflection on future challenges, in: Journal of Manufacturing Technology Management, Jg. 20, Nr. 5, S. 547-567.

Bauer, W./Schlund, S./Marrenbach, D./Ganschar, O. (2014): Industrie 4.0 - Volkswirtschaftliches Potenzial für Deutschland, BITKOM Bundesverband Informationswirtschaft, Telekommunikation und neue Medien e.V. /Fraunhofer-Institut für Arbeitswirtschaft und Organisation IAO, Berlin/Stuttgart.

Beckenbauer, B. (2006): Internationales Management industrieller Dienstleistungen, St. Gallen.

Belz, C./Schuh, G./Groos, A./Reinecke, S. (1997): Industrie als Dienstleister, St. Gallen.

Borgmeier, A. (2002): Teleservice im Maschinen- und Anlagenbau: Anwendungen und Gestaltungsempfehlungen, Wiesbaden.

Boyt, T./Harvey, M. (1997): Classification of Industrial Services - A Model with Strategic Implications, in: Industrial Marketing Management, Jg. 26, Nr. 4, S. 291-300.

Brady, T./Davies, A./Gann, D. (2005): Creating value by delivering integrated solutions, in: International Journal of Project Management, Jg. 23, Nr. 5, S. 360-365.

Buschak, D./Lerch, C./Gotsch, M. (2014): Messung des Service Value innovativer Dienstleistungen in der Industrie - Eine integrierte Anbieter-Kunden-Perspektive, in: Bruhn, M./Hadwich, K. (Hrsg.): Service Value als Werttreiber - Konzepte, Messung und Steuerung, Wiesbaden, S. 91-113.

Engelhardt, W./Kleinaltenkamp, M./Reckenfelderbäumer, M. (1993): Leistungsbündel als Absatzobjekte: Ein Ansatz zur Überwindung der Dichotomie von Sach- und Dienstleistungen, in: Zeitschrift für betriebswirtschaftliche Forschung, 45. Jg., Nr. 5, S. 395-426.

Erramilli, M. (1989): Entry Mode Choice in Service Industries, in: International Marketing Review, Jg. 7, Nr. 5/6, S. 50-62.

Gebauer, H./Bravo-Sanchez, C./Fleisch, E. (2008): Service strategies in product manufacturing companies, in: Business Strategy Series, Jg. 9, Nr. 1, S. 12-20.

Gebauer, H./Fleisch, E./Friedli, T. (2005): Overcoming the Service Paradox in Manufacturing Companies, in: European Management Journal, Jg. 23, Nr. 1, S. 14-26.

Gebauer, H. (2004): Die Transformation vom Produzenten zum produzierenden Dienstleister, St. Gallen. 
Gerhard, A. (2000): Produktbegleitende Dienstleistungen: Neue Herausforderungen an den Maschinenbau, in: Technologie und Management, 49, S. 26-27.

Kirsch, B. (2004): Entwicklung und kooperative Nutzung eines modernen Teleservice-Systems, in: Meier, H./Schramm, J. (Hrsg.): Dienstleistungsorientierte Geschäftsmodelle im Maschinen- und Anlagenbau - Vom Basisangebot zum Betreibermodell, Berlin, S. 303-323.

Knight, G. (1999): International services marketing: review of research 1980-1998, in: Journal of Services Marketing, Jg. 13, Nr. 4/5, S. 347-360.

Lay, G. (2014): Servitization of Industry: Origins and Definitions, in: Lay, G. (Hrsg.): Servitization in Industry, Heidelberg u.a., S. 1-20.

Lay, G./Rainfurth, C. (2001): Aktionsfelder für das Service Management in der Investitionsgüterindustrie, in: Industrie Management, Jg. 17, Nr. 2, S. 9-12.

Mathieu, V. (2001): Product services: from a service supporting the product to a service supporting the client, in: Journal of Business \& Industrial Marketing, Jg. 16, Nr. 1, S. 39-58.

Matthyssens, P./Vandenbempt, K. (2008): Moving from basic offerings to value-added solutions: Strategies, barriers and alignment, in: Industrial Marketing Management, Jg. 37, Nr. 3, S. 316-328.

Matthyssens, P./Vandenbempt, K. (2010): Service addition as business market strategy: identification of transition trajectories, in: Journal of Service Management, Jg. 21, Nr. 5, S. 693-714.

Meyer, A./Blümelhuber, C. (1998): Dienstleistungs-Innovation, in: Meyer, A. (Hrsg.): Handbuch Dienstleistungsmarketing, Band 1, Stuttgart, S. 807-826.

More, R. (2001): Creating profits from integrated product-service strategies, in: Ivey Business Journal, Jg. 65, Nr. 5, S. 75-81.

Münster, M./Meiren, T. (2011): Internet-basierte Services im Maschinen- und Anlagenbau, Stuttgart.

Oliva, R./Kallenberg, R. (2003): Managing the transition from products to services, in: International Journal of Service Industry Management, Jg. 14, Nr. 2, S. 160-172.

Penttinen, E./Palmer, J. (2007): Improving firm positioning through enhanced offerings and buyerseller relationships, in: Industrial Marketing Management, Jg. 36, Nr. 5, S. 552-564.

Schniering, N. (2009): Industrielle Dienstleistungsinnovationen - Eine praxisorientierte Analyse der Determinanten und Erfolgswirkungen, Münster.

Schröter, M./Biege, S./Lerch, C. (2008): Dienstleistungsbasierte Geschäftsmodelle für die Montage, in: Witte, K.-W./Vielhaber, W. (Hrsg.): Lebenszyklusoptimierte Montage - Modulare Systeme und neue Geschäftsmodelle, Aachen, S. 39-77.

Spath, D./Demuß, L. (2003): Entwicklung hybrider Produkte - Gestaltung materieller und immaterieller Leistungsbündel, in: Bullinger, H./Scheer, A. (Hrsg.): Service Engineering - Entwicklung und Gestaltung innovativer Dienstleistungen, Heidelberg u.a., S. 467-506.

Spath, D./Ganschar, O./Gerlach, S./Hämmerle, M./Krause, T./Schlund, S. (2013): Produktionsarbeit der Zukunft - Industrie 4.0, Fraunhofer Institut für Arbeitswirtschaft und Organisation IAO, Stuttgart.

Tukker, A. (2004): Eight types of product-service-systems: Eight ways to substainability? Experiences from Suspronet, in: Business Strategy and the Environment, Jg. 13, Nr. 4, S. 246-260.

Vandermerwe, S./Chadwick, M. (1989): The Internationalization of Services, in: The Services Industries Journal, Jg. 9, Nr. 1, S. 79-93.

Vandermerwe, S./Rada, J. (1988): Servitization of Business: Adding Value by Adding Services, in: European Management Journal, Jg. 6, Nr. 4, S. 314-324. 
Velamuri, V./Neyer, A./Möslein, K. (2011): Hybrid value creation: a systematic review of an evolving research area, in: Journal für Betriebswirtschaft, Jg. 61, Nr. 1, S. 3-35.

Voeth, M./Bertels, V. (2014): Service Value von produktbegleitenden Dienstleistungen, in: Bruhn, M./Hadwich, K. (Hrsg.): Service Value als Werttreiber - Konzepte, Messung und Steuerung, Wiesbaden, S. 283-300.

Wassermann, R. (2010): Internationalisierung mit produktbegleitenden Dienstleistungen und hybriden Produkten - Eine pfadorientierte Analyse am Beispiel deutscher Maschinenbauunternehmungen, Heidelberg.

Wise, R./Baumgartner, P. (1999): Go Downstream - The New Profit Imperative in Manufacturing, in: Harvard Business Review, Jg. 77, Nr. 5, S. 133-141.

Christian Lerch, Dipl. rer. pol. techn., ist Leiter des Geschäftsfelds „Industrielle Dienstleistungen“ im Competence Center Industrie- und Serviceinnovationen am Fraunhofer-Institut für System- und Innovationsforschung ISI in Karlsruhe.

Anschrift: Fraunhofer-Institut für System- und Innovationsforschung ISI, Breslauer Straße 48, D-76139 Karlsruhe, Tel.: +49 (0)721/6809-386, Fax: +49 (0)721/6809-152, E-Mail: christian.lerch@isi.fraunhofer.de

Matthias Gotsch, Dr., ist Projektleiter im Competence Center Industrie- und Serviceinnovationen am Fraunhofer-Institut für System- und Innovationsforschung ISI in Karlsruhe.

Anschrift: Fraunhofer-Institut für System- und Innovationsforschung ISI, Breslauer Straße 48, D-76139 Karlsruhe, Telefon +49 (0)721/6809-325, Fax +49 (0)721/6809-77325, EMail: matthias.gotsch@isi.fraunhofer.de 